INTERDISCIPLINARIA ARCHAEOLOGICA

News and views

\title{
Survey of Mural Pigment Samples from the Rotunda of Saint Catherine, Znojmo, Czech Republic
}

\author{
Kateřina Dvořákováa ${ }^{*}$ \\ aPrivate professional expert, Absolonova 73, 62400 Brno, Czech Republic
}

\section{ARTICLE INFO}

\section{Article history:}

Received: 16 May 2011

Accepted: 20 December 2011

\section{Keywords:}

Romanesque painting

pigments

laboratory survey

interpretation

analysis

dating

\section{$A B S T R A C T$}

The article looks at the evaluation of dating of pigment samples taken from murals in the Rotunda of Saint Catherine in Znojmo, Czech Republic (see Merhautová, Třeštík 1984). The samples were taken on July $11^{\text {th }}, 2006$ by Petr Kadlec, a restorer and academically trained painter, for the South Moravia Region Art and Monument Preservation Department.

\section{Introduction}

The investigations of pigments in the Rotunda of St. Catherine in Znojmo were not finished during the $20^{\text {th }}$ century due to the lack of interest and financial resources. As a result a new, the seventh, sampling and survey of these extraordinary murals was organized.

\section{Theory}

\subsection{Brief history of the sampling of the murals}

Taking a brief look into the history of sampling of the murals; the National Institute of Monument and Nature Preservation in Prague made its first pigment analysis in 1967 (under Ing. Šedivý), following the process of the restorers Alt, Lorek and Martan. The second sampling, using the same procedure, was conducted on May $25^{\text {th }}, 1971$, also by Ing. Šedivý. The third was taken on May $26^{\text {th }}, 1971$. The fourth sampling was taken after the conclusion of the restoration of five samples (H. Králová on basis of microscopic survey, microchemical tests, emission spectrography). The samples were taken

"Corresponding author. E-mail: k-dvorakova@volny.cz from unspecified parts of the mural. The fifth sampling, evaluated on July $24^{\text {th }}$, 1986 , referred to another five samples (H. Králová).

The sixth sampling underwent preliminary evaluation Jan $31^{\text {st }}, 1992$ (Geoarch, Prague, Zeman, Růžičková). According to the preliminary report the samples were only a few $\mathrm{mm}^{3}$. They were marked (M) and taken by Mr. Martan before 1990, as well as after 1990 (MZR). The work procedure during the laboratory evaluation was as follows:

- Scanning on raster microscope JXA50A.

- Substance analysis on energy-dispersive analyzer EDAX.

As mentioned in the report it was impossible to make final conclusions: "Even though the results show that in the mixture of pigments there are elements which should not be present in medieval pigments". The sampling from 2006 is the seventh in the history of sampling of these murals.

\section{Materials and Methods}

The samples of the mural were taken on July $11^{\text {th }}, 2006$, numbered 1-23, and submitted to a laboratory survey (Figure 1). 


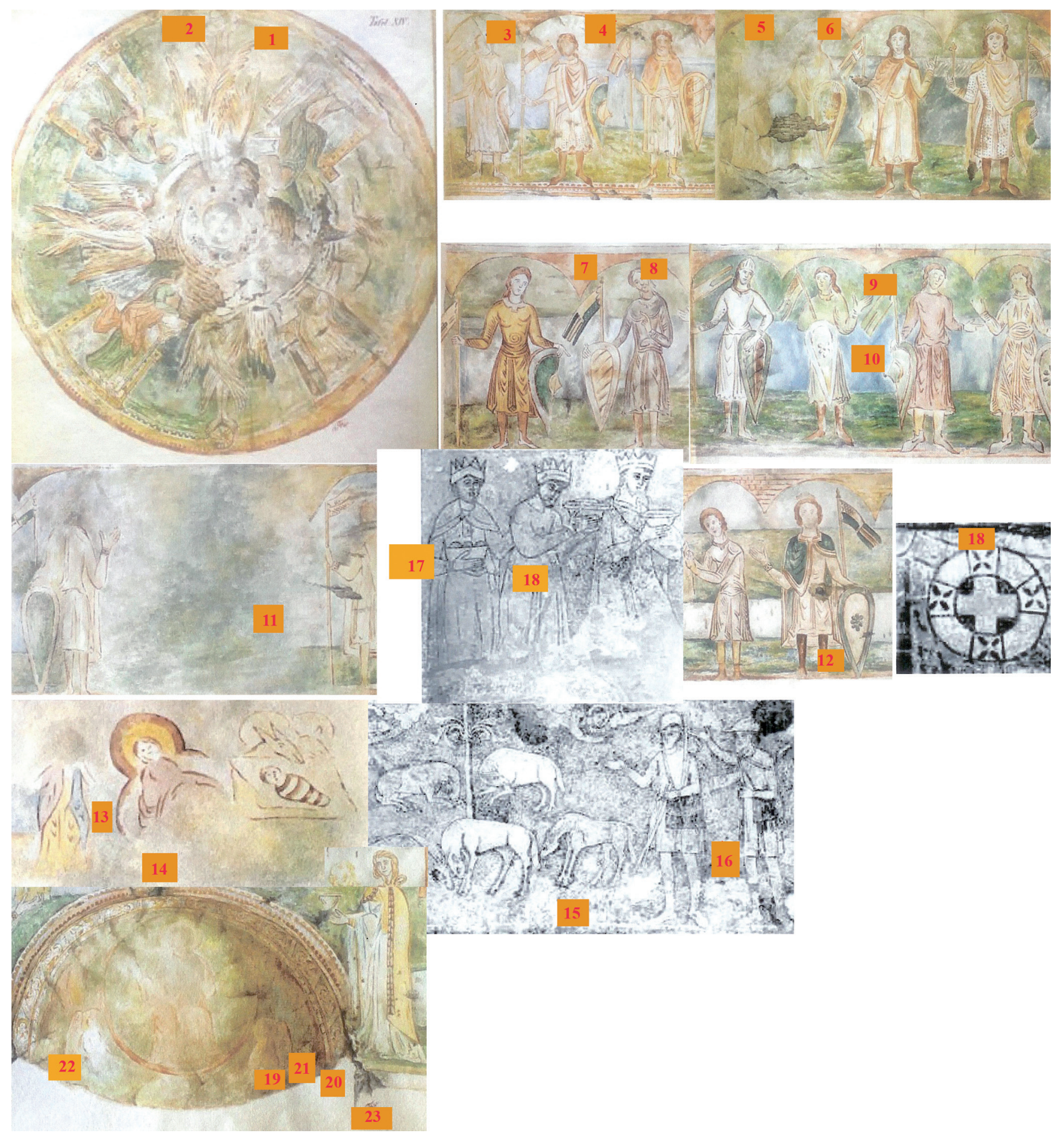

Figure 1. Location of samples, which were used for analysis (drawing M. Trapp, 1862, photo authors' archive).

After the preparation of cross sections, and casting into Spofakryl resin, the samples were polished and photographed in colour. After measuring and describing the respective layers of the samples, microscopic and microchemical analysis was conducted. Element analysis was done with an electron microanalyzer (JXA 50A/EDAX). Histochemical staining with fuchsine $\mathrm{S}$ was applied on polished slice of casted samples (the proteins get pink or purple-red colour). The presence of oils was confirmed with a foam test.
An electron microanalyzer is suitable for identification of elements, or oxides in specific layers of the paint. This nondestructive analysis is conducted on a slice of the casted sample, which has a vacuum evaporated carbon layer.

It's possible to analyse a point or place that is marked by a coordinate point. The programme equipment of the analyzer makes it possible to determine which element responds to the pica in RTG illumination and by subtraction of their intensity it is possible to establish their quantitative rates. 
For the interpretation of results it's important to know the structure of the pigments in a given colour layer. Microscopic and microchemical analyses were conducted on all samples. The results of the measurement by electron microanalyzer (evaluated in the form of oxides) are shown in the tables and they are part of the sampling documentation.

\section{Results}

The presence of following elements was confirmed in the samples by microscopical and microchemical analysis:

- Calcium carbonate: proof - dissolves in dilute mineral acids with release of $\mathrm{CO}_{2}$. Test for presence of $\mathrm{Ca}^{2+}$ (by applying $2 \mathrm{~N} \mathrm{HCl}$ crystals of $\mathrm{CaCl}_{2}$ are created, when distilled $\mathrm{H}_{2} \mathrm{O}$ with $2 \mathrm{~N} \mathrm{H}_{2} \mathrm{SO}_{4}$ is applied, after warming up needle-like crystals are formed).

- Calcium sulphate: proof - dissolves in dilute mineral acids with release of $\mathrm{CO} 2$. Test for presence of $\mathrm{Ca}^{2+}$.

- Lead white: homogenous, very fine, round, anisotropic, birefringent element, dissolves in dilute mineral acids with release of $\mathrm{CO}_{2}$. Test for presence of $\mathrm{Pb}^{2+}$ (by application of $2 \mathrm{~N} \mathrm{HNO}_{3}$ characteristic crystals of $\mathrm{Pb}\left(\mathrm{NO}_{3}\right)_{2}$ are formed after warming up, after the addition of $\left(\mathrm{CH}_{3} \mathrm{COOH}\right)_{2} \mathrm{Cu}, \mathrm{CH}_{3} \mathrm{COOHN}$ solution and $\mathrm{CH}_{3} \mathrm{COOH}$ and crystal of $\mathrm{KNO}_{2}$ dark brown cubic crystals of $\mathrm{K}_{2} \mathrm{PbCu}\left(\mathrm{NO}_{2}\right)_{6}$ are formed with presence of $\mathrm{Pb}^{2+}$.

- Zinc white: typical aspect and fluorescence colour, confirmed by element analysis.

- Titanium white - typical aspect and fluorescence colour, confirmed by element analysis.

- Ochres: heterogeneous, isotropic, contain, highly birefringent colourless elements of flint. They dissolve in concentrated mineral acids. Test for presence of $\mathrm{Fe}^{3+}$ (the sample is transformed into solution, $2 \mathrm{~N} \mathrm{HCl}$ and crystals of $\mathrm{NH}_{4} \mathrm{SCN}$ are added, in presence of $\mathrm{Fe}^{3+}$ a blood red colouration is visible).

- Iron oxide red (red ochres, clays): inhomogeneous, anisotropic element, of various shape, colour, structure and size. They resist to alkalis, they dissolve only in hot acids. Test for presence of $\mathrm{Fe}^{3+}$.

- Synthetic iron oxide reds have the same qualities as the natural ones. The pigment does not have characteristic microscopical qualities. The distinction between the finest natural oxides and the synthetic ones is very difficult.

- Mars yellow (synthetically created iron oxide yellow): fine homogeneous elements, chemically resistant to acids and alkalis. Test for presence of $\mathrm{Fe}^{3+}$.

- Green earth (green clay): glaucous, slightly birefringent elements of round shape, partially soluble in acids and alkalis, after being warmed up they get brown. Test for presence of $\mathrm{Fe}^{3+}$. (To the acidized solution of the sample a drop of $2 \%$ solution a, $\mathrm{a}^{6}$ - dipyridyl, the surfactant reacts with $\mathrm{Fe}^{2+}$ and a dark red adduct is produced).

- Synthetic ultramarine: fine blue and round shaped isotropic elements which resist to the heat and alkali treatment.
- Minium: elements of various sizes slightly birefringent in transiting light, soluble in dilute mineral acid, not soluble in an alkali. Positive test for presence of $\mathrm{Pb}^{2+}$

- Transparent chromium oxide: bright green elements, slightly round, birefringent, resistant to acids and alkalis. Chrome confirmed by elements analysis.

- Schweinfurt green (Paris green, Emerald green), complex of octane and cupric arsenite. Small, regular and round elements, soluble in mineral acids. During alkali treatment in heat, the elements do decompose and blue $\mathrm{Cu}(\mathrm{OH})_{2}$ is formed. Copper and arsenic are confirmed by element analysis.

\section{Rendering}

Bright ochre, rough grained, lime sand rendering contains calcium carbonate, granules of silicon, red pieces of natural ochres and carbon fragments. It had been prepared with lime, milled calcium carbonate, hydraulic additives (sand, rubble and crushed carbon), that improve volume stability, mechanical strength and stiffness; they improve the resistance to water and frost. In some of the formulas (unoriginal) only the white layer of calcium carbonate is noted.

\section{Painting}

The paint layers are laid directly on the plaster layer, without any oil or dirt layers. The painting layers contain the respective pigment(s), calcium sulphate, clays and phosphorus. The presence of phosphorus and positive reaction for the presence of proteins show that the casein binder was used in these paint layers. At the same time it could also be sodium phosphate, used as an additive to slows the setting of gypsum and that was used in upper layers of the mural.

None of the samples showed a positive reaction to the presence of oils. Samples No. 13, 16, 19 and 23 were analyzed only microscopically and microchemically (due to fact that already known characteristics would be confirmed). The results of all analyses are noted in the figures of respective painting layers of the respective samples and are mentioned in the photo documentation.

\section{Discussion}

Up to now the professional literature has asserted that during recent restoration the murals were restored to their original conditions and do not have any overpaintings, newer additions, nor unoriginal pigments. The pigments used should, according to professional opinions, correspond to medieval pigments.

In this investigation the laboratory analyses of samples concluded that the majority of the murals contain pigments which cannot be present in a Romanesque painting.

- Sample 1 (Figure 2): green colour - green earth, chrome oxide, barium sulphate, colouring earth, calcium sulphate.

- Sample 2 (Figure 3): green colour - chrome oxide, barium sulphate, colouring earth, calcium sulphate.

- Sample 3 (Figure 4): red colour - red ochre, colouring earth, calcium sulphate. 

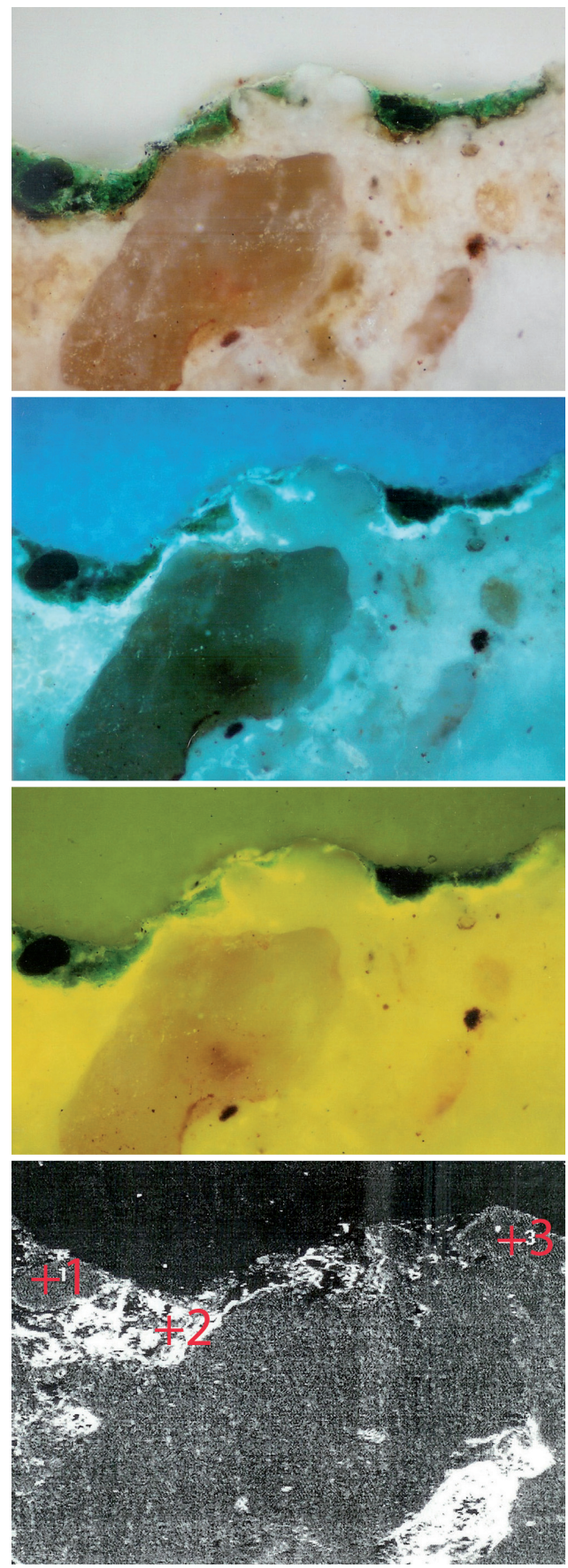

\begin{tabular}{cccccc}
\hline & $\mathbf{1}$ & & \multicolumn{2}{c}{$\mathbf{2}$} & \\
Oxide & Wt [\%] & Oxide & Wt [\%] & Oxide & Wt [\%] \\
\hline $\mathrm{MgO}$ & 5.54 & $\mathrm{Na}_{2} \mathrm{O}$ & 0.44 & $\mathrm{MgO}$ & 2.99 \\
$\mathrm{Al}_{2} \mathrm{O}_{3}$ & 7.38 & $\mathrm{MgO}$ & 2.77 & $\mathrm{SiO}_{2}$ & 11.08 \\
$\mathrm{SiO}_{2}$ & 52.02 & $\mathrm{Al}_{2} \mathrm{O}_{3}$ & 5.39 & $\mathrm{SO}_{3}$ & 1.82 \\
$\mathrm{~K}_{2} \mathrm{O}$ & 9.65 & $\mathrm{SiO}_{2}$ & 33.3 & $\mathrm{CaO}$ & 84.12 \\
$\mathrm{Fe}_{2} \mathrm{O}_{3}$ & 25.4 & $\mathrm{SO}_{3}$ & 13.63 & & \\
& & $\mathrm{~K}_{2} \mathrm{O}$ & 6.14 & & \\
& & $\mathrm{CaO}$ & 3.92 & & \\
& & $\mathrm{BaO}$ & 15.34 & & \\
& & $\mathrm{Cr}_{2} \mathrm{O}_{3}$ & 5.01 & & \\
& & $\mathrm{Fe}_{2} \mathrm{O}_{3}$ & 14.05 & & \\
Total & 100 & $\mathrm{Total}$ & 100 & Total & 100 \\
\hline
\end{tabular}

Figure 2. Sample 1; green colour - green earth, chrome oxide, barium sulphate, colouring earth, calcium sulphate.

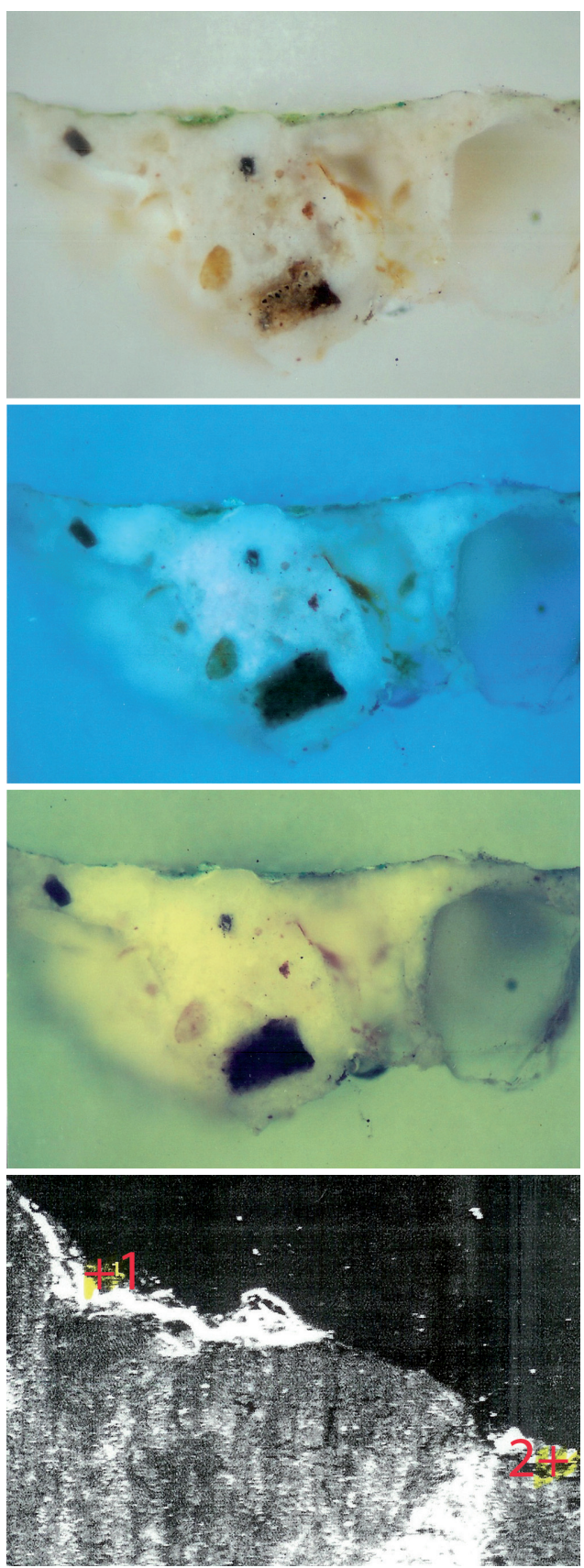

\begin{tabular}{ccc}
\hline & $\mathbf{1}$ & $\mathbf{2}$ \\
Oxide & $\mathbf{W t}[\boldsymbol{\%}]$ & $\mathbf{W t}[\mathbf{\%}]$ \\
\hline $\mathrm{Na}_{2} \mathrm{O}$ & 0.59 & 2.74 \\
$\mathrm{MgO}$ & 0.55 & 2.33 \\
$\mathrm{Al}_{2} \mathrm{O}_{3}$ & 4.3 & 3.58 \\
$\mathrm{SiO}_{2}$ & 25.7 & 26.06 \\
$\mathrm{SO}_{3}$ & 21.84 & 17.86 \\
$\mathrm{~K}_{2} \mathrm{O}$ & 3.14 & 5.17 \\
$\mathrm{CaO}$ & 13.52 & 4.58 \\
$\mathrm{BaO}$ & 12.99 & 15.61 \\
$\mathrm{Cr}_{2} \mathrm{O}_{3}$ & 4.98 & 10.67 \\
$\mathrm{Fe}_{2} \mathrm{O}_{3}$ & 12.38 & 11.41 \\
$\mathrm{Total}$ & 100 & 100 \\
\hline
\end{tabular}

Figure 3. Sample 2; green colour - chrome oxide, barium sulphate, colouring earth, calcium sulphate. 

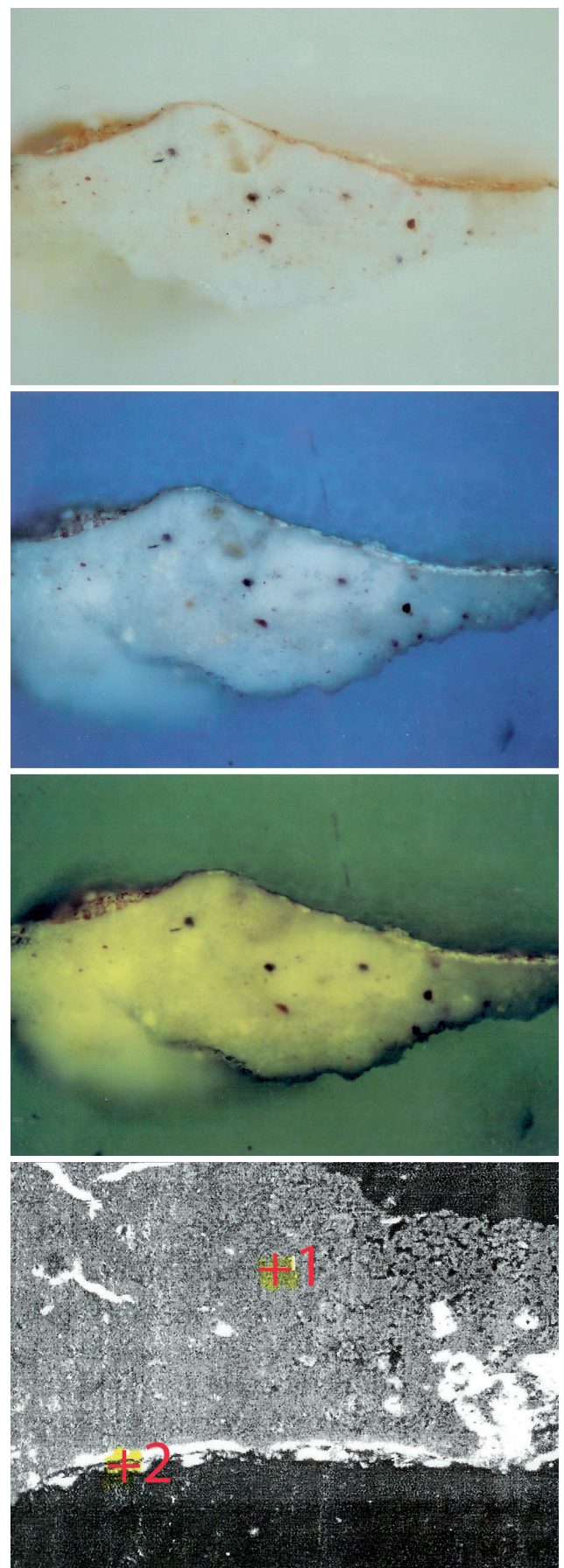

\begin{tabular}{cccc}
\hline & $\mathbf{1}$ & & \\
Oxide & Wt [\%] & Oxide & Wt [\%] \\
\hline $\mathrm{CaCO}_{3}$ & & $\mathrm{Na}_{2} \mathrm{O}$ & 1.86 \\
& $\mathrm{MgO}$ & 0.58 \\
& & $\mathrm{Al}_{2} \mathrm{O}_{3}$ & 8.41 \\
& $\mathrm{SiO}_{2}$ & 7.07 \\
& $\mathrm{SO}_{3}$ & 4.87 \\
& $\mathrm{~K}_{2} \mathrm{O}$ & 1.17 \\
& $\mathrm{CaO}$ & 26.78 \\
& $\mathrm{TiO}_{2}$ & 9.19 \\
& & $\mathrm{Fe}_{2} \mathrm{O}_{3}$ & 40.06 \\
& $\mathrm{Total}^{\text {Total }}$ & & 100 \\
\hline
\end{tabular}

Figure 4. Sample 3; red colour - red ochre, colouring earth, calcium sulphate.
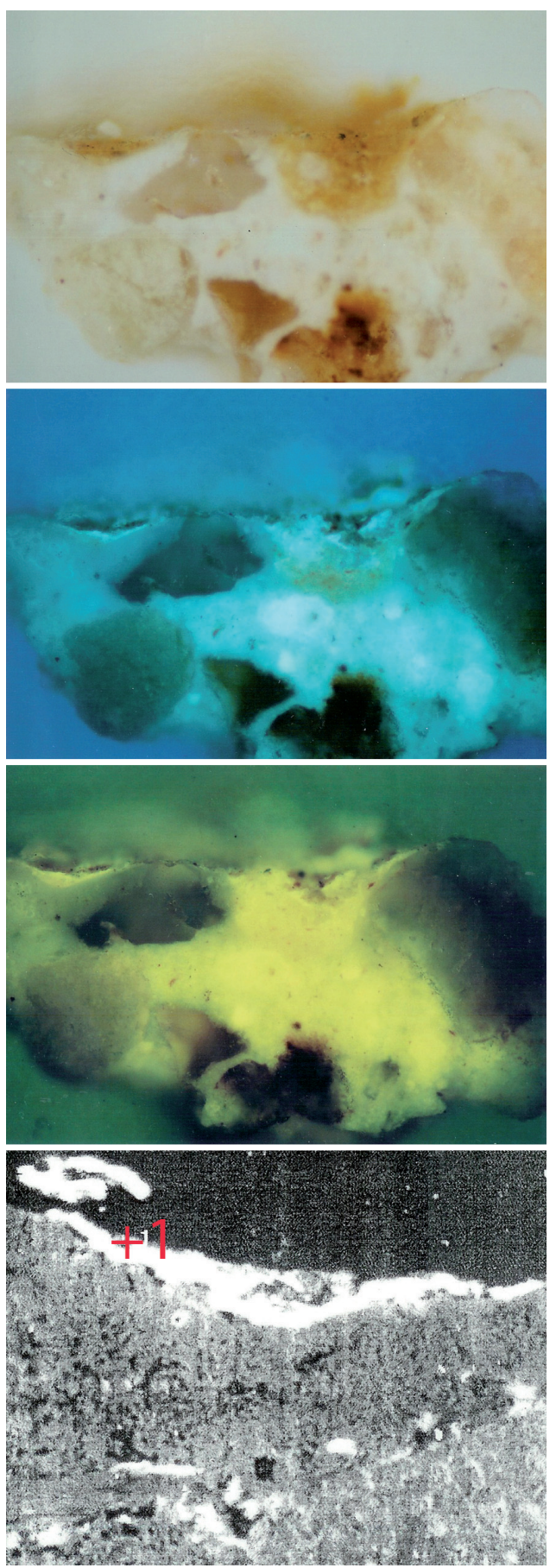

\begin{tabular}{cc}
\hline & $\mathbf{1}$ \\
Oxide & Wt [\%] \\
\hline $\mathrm{Al}_{2} \mathrm{O}_{3}$ & 17.87 \\
$\mathrm{SiO}_{2}$ & 23.77 \\
$\mathrm{SO}_{3}$ & 9.15 \\
$\mathrm{~K}_{2} \mathrm{O}$ & 0.97 \\
$\mathrm{CaO}$ & 4.21 \\
$\mathrm{Fe}_{2} \mathrm{O}_{3}$ & 44.03 \\
Total & 100
\end{tabular}

Figure 5. Sample 4; yellow colour - mars yellow, colouring earth, calcium sulphate. 

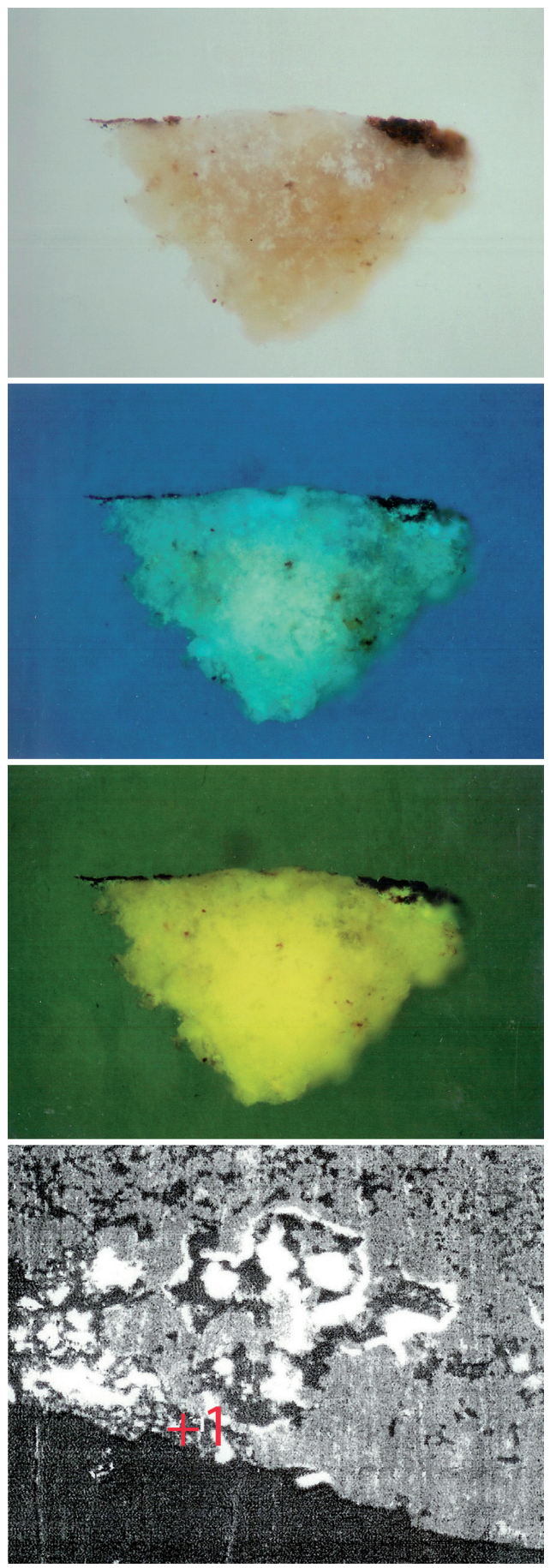

\begin{tabular}{cc}
\hline & $\mathbf{1}$ \\
Oxide & Wt [\%] \\
\hline $\mathrm{MgO}$ & 1.07 \\
$\mathrm{Al}_{2} \mathrm{O}_{3}$ & 2.26 \\
$\mathrm{SiO}_{2}$ & 10.62 \\
$\mathrm{SO}_{3}$ & 6.85 \\
$\mathrm{~K}_{2} \mathrm{O}$ & 0.58 \\
$\mathrm{CaO}$ & 4.08 \\
$\mathrm{Fe}_{2} \mathrm{O}_{3}$ & 59.4 \\
$\mathrm{ZnO}$ & 15.15 \\
$\mathrm{Total}$ & 100 \\
\hline
\end{tabular}

Figure 6. Sample 5; red colour - red ochre, colouring earth, calcium sulphate, zinc white.

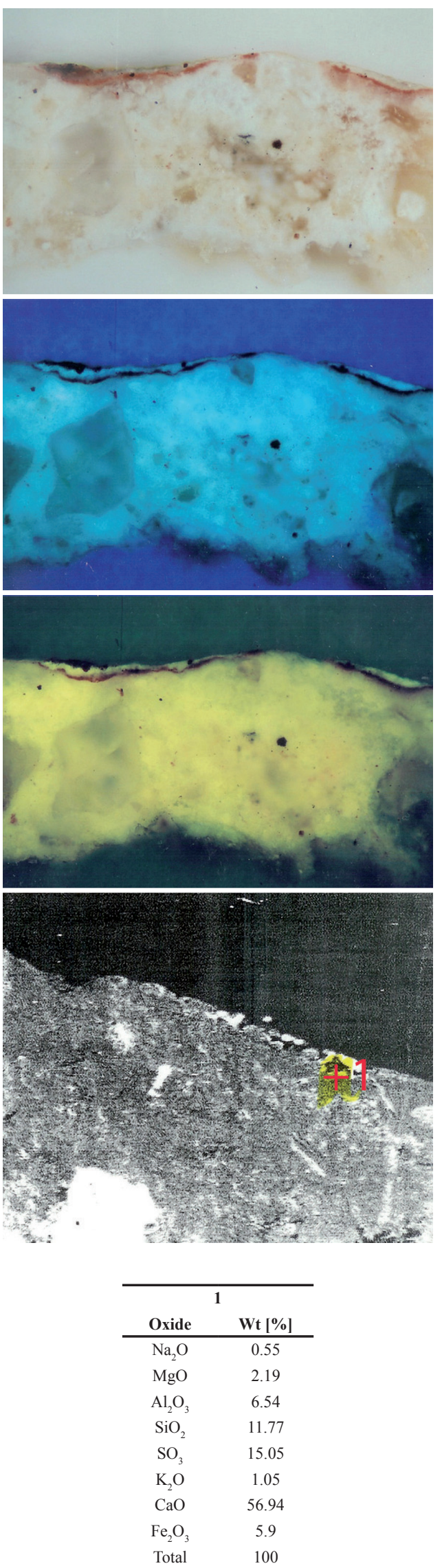

Figure 7. Sample 6; red colour - red ochre, colouring earth, calcium sulphate. 

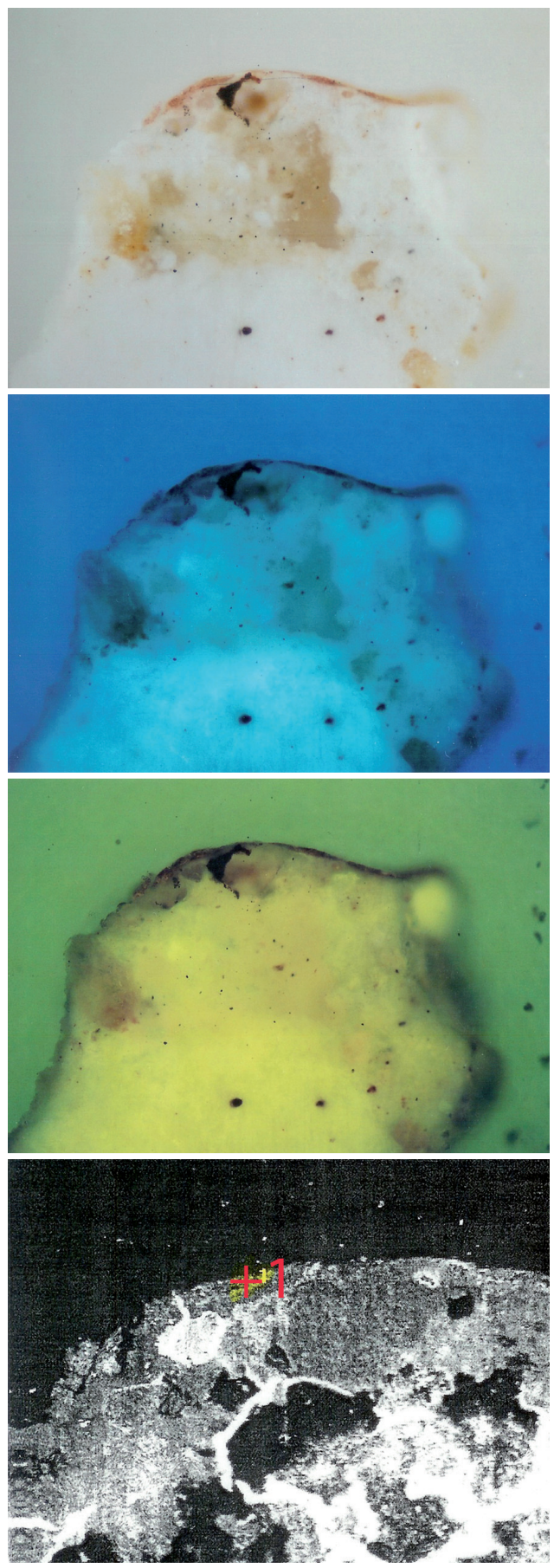

\begin{tabular}{cc}
\hline Oxide & Wt [\%] \\
\hline $\mathrm{Na}_{2} \mathrm{O}$ & 0.71 \\
$\mathrm{MgO}$ & 0.77 \\
$\mathrm{Al}_{2} \mathrm{O}_{3}$ & 3.56 \\
$\mathrm{SiO}_{2}$ & 3.61 \\
$\mathrm{SO}_{3}$ & 40.2 \\
$\mathrm{~K}_{2} \mathrm{O}$ & 0.31 \\
$\mathrm{CaO}$ & 35.57 \\
$\mathrm{TiO}_{2}$ & 3.03 \\
$\mathrm{Fe}_{2} \mathrm{O}_{3}$ & 12.24 \\
$\mathrm{Total}$ & 100 \\
\hline
\end{tabular}

Figure 8. Sample 7; red colour - red ochre, colouring earth, calcium sulphate.
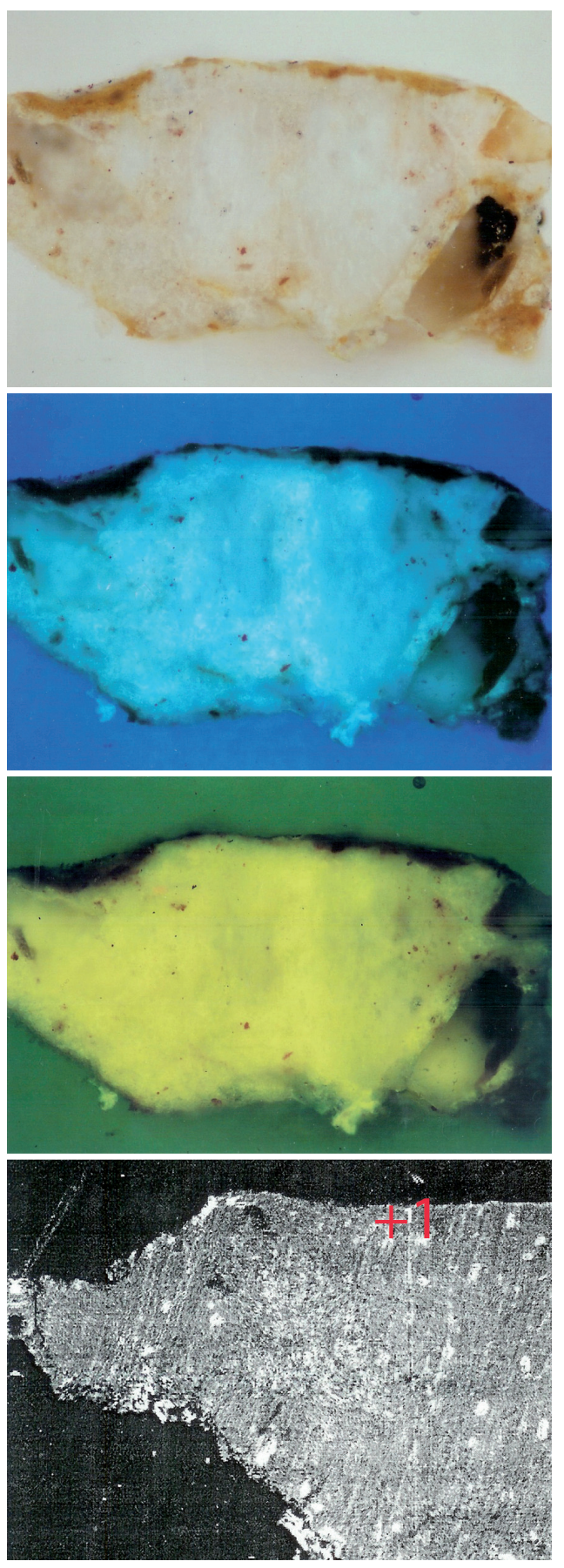

\begin{tabular}{cc}
\hline & $\mathbf{1}$ \\
Oxide & Wt [\%] \\
\hline $\mathrm{MgO}$ & 5.22 \\
$\mathrm{Al}_{2} \mathrm{O}_{3}$ & 15.32 \\
$\mathrm{SiO}_{2}$ & 39.77 \\
$\mathrm{P}_{2} \mathrm{O}_{5}$ & 3.56 \\
$\mathrm{SO}_{3}$ & 3.48 \\
$\mathrm{~K}_{2} \mathrm{O}$ & 2.89 \\
$\mathrm{CaO}$ & 18.78 \\
$\mathrm{TiO}_{2}$ & 0.73 \\
$\mathrm{Fe}_{2} \mathrm{O}_{3}$ & 10.26 \\
$\mathrm{Total}$ & 100
\end{tabular}

Figure 9. Sample 8; yellow colour - yellow and red ochres, colouring earth 

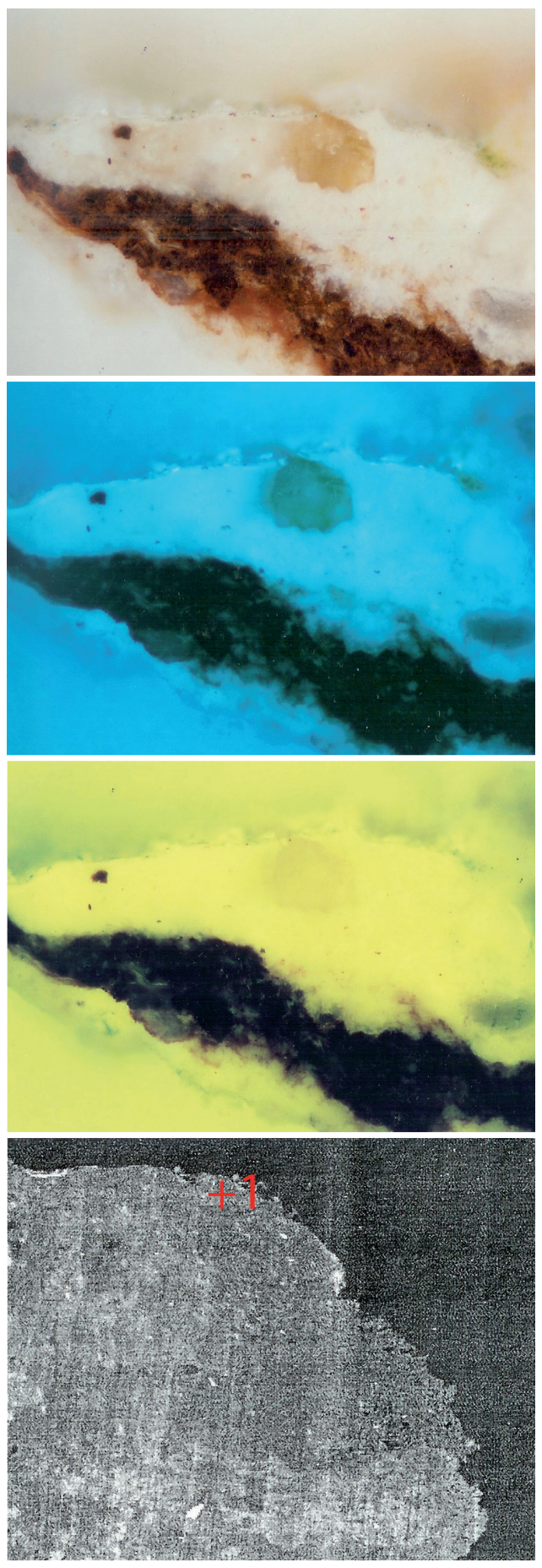

\begin{tabular}{cc}
\hline & $\mathbf{1}$ \\
Oxide & Wt [\%] \\
\hline $\mathrm{MgO}$ & 3.76 \\
$\mathrm{Al}_{2} \mathrm{O}_{3}$ & 7.25 \\
$\mathrm{SiO}_{2}$ & 39.77 \\
$\mathrm{SO}_{3}$ & 14.72 \\
$\mathrm{~K}_{2} \mathrm{O}$ & 6.13 \\
$\mathrm{CaO}$ & 7.88 \\
$\mathrm{BaO}$ & 7.68 \\
$\mathrm{Fe}_{2} \mathrm{O}_{3}$ & 12.81 \\
$\mathrm{Total}$ & 100 \\
\hline
\end{tabular}

Figure 10. Sample 9; green colour - green earth, chrome oxide (?), barium sulphate, colouring earth, ochres, calcium sulphate.
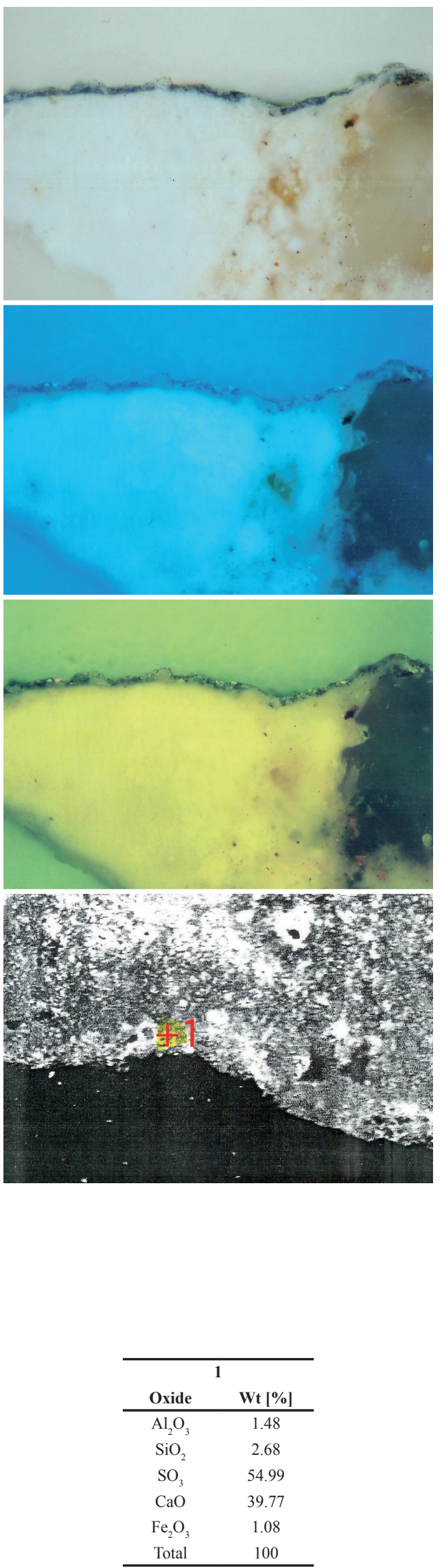

Figure 11. Sample 10; blue colour - artificial ultramarine, colouring earth, ochres, calcium sulphate. 

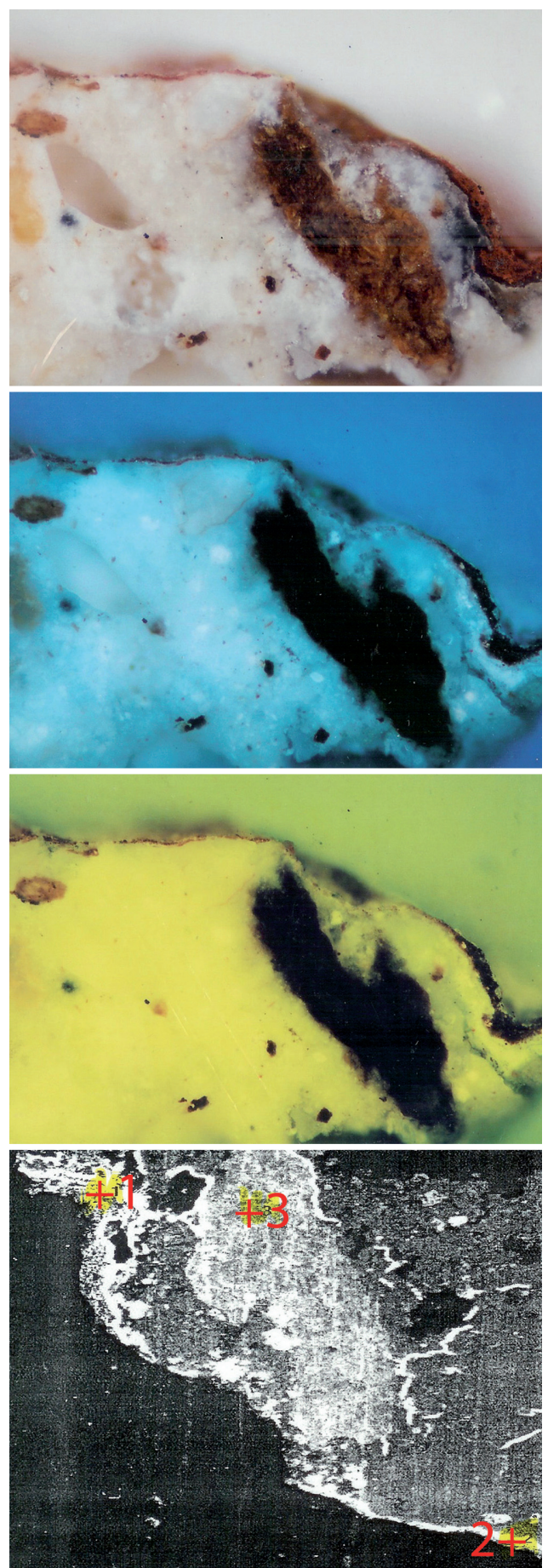

\begin{tabular}{cccccc}
\hline & \multicolumn{2}{c}{$\mathbf{1}$} & \multicolumn{2}{c}{$\mathbf{2}$} & \multicolumn{2}{c}{$\mathbf{3}$} \\
Oxide & $\mathbf{W t}$ [\%] & Oxide & $\mathbf{W t ~ [ \% ] ~}$ & Oxide & Wt [\%] \\
\hline $\mathrm{Al}_{2} \mathrm{O}_{3}$ & 4.57 & $\mathrm{MgO}$ & 0.91 & $\mathrm{MgO}$ & 2.05 \\
$\mathrm{SiO}_{2}$ & 1.57 & $\mathrm{Al}_{2} \mathrm{O}_{3}$ & 12.63 & $\mathrm{Al}_{2} \mathrm{O}_{3}$ & 22.91 \\
$\mathrm{PbO}_{2}$ & 37.8 & $\mathrm{Si}_{2}$ & 6.27 & $\mathrm{SiO}_{2}$ & 34.24 \\
$\mathrm{CaO}$ & 3.51 & $\mathrm{SO}_{3}$ & 18.33 & $\mathrm{SO}_{3}$ & 1.7 \\
$\mathrm{Cr}_{2} \mathrm{O}_{3}$ & 4.16 & $\mathrm{CaO}$ & 19.1 & $\mathrm{~K}_{2} \mathrm{O}$ & 3.8 \\
$\mathrm{Fe}_{2} \mathrm{O}_{3}$ & 48.4 & $\mathrm{TiO}_{2}$ & 3.84 & $\mathrm{CaO}$ & 1.01 \\
& & $\mathrm{Fe}_{2} \mathrm{O}_{3}$ & 38.93 & $\mathrm{Fe}_{2} \mathrm{O}_{3}$ & 34.29 \\
Total & 100 & Total & 100 & Total & 100 \\
\hline
\end{tabular}

Figure 12. Sample 11; I - coating, red colour - red ochre, colouring earth, calcium sulphate; II - lower coating, red colour - red ochre, colouring earth, cinnabar, chrome.
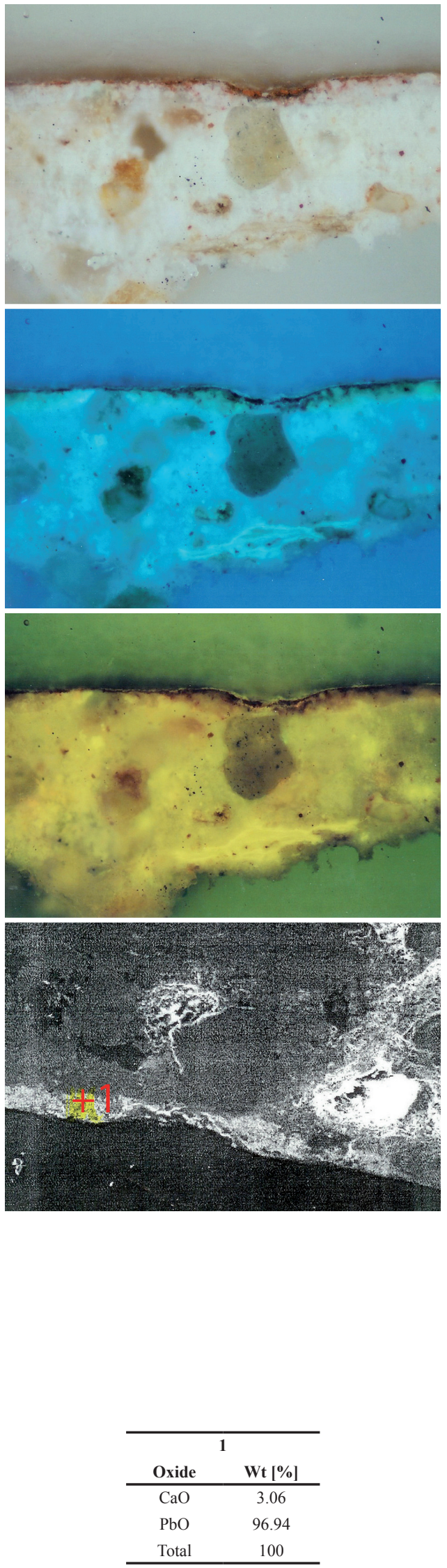

Figure 13. Sample 12; red colour - cinnabar. 

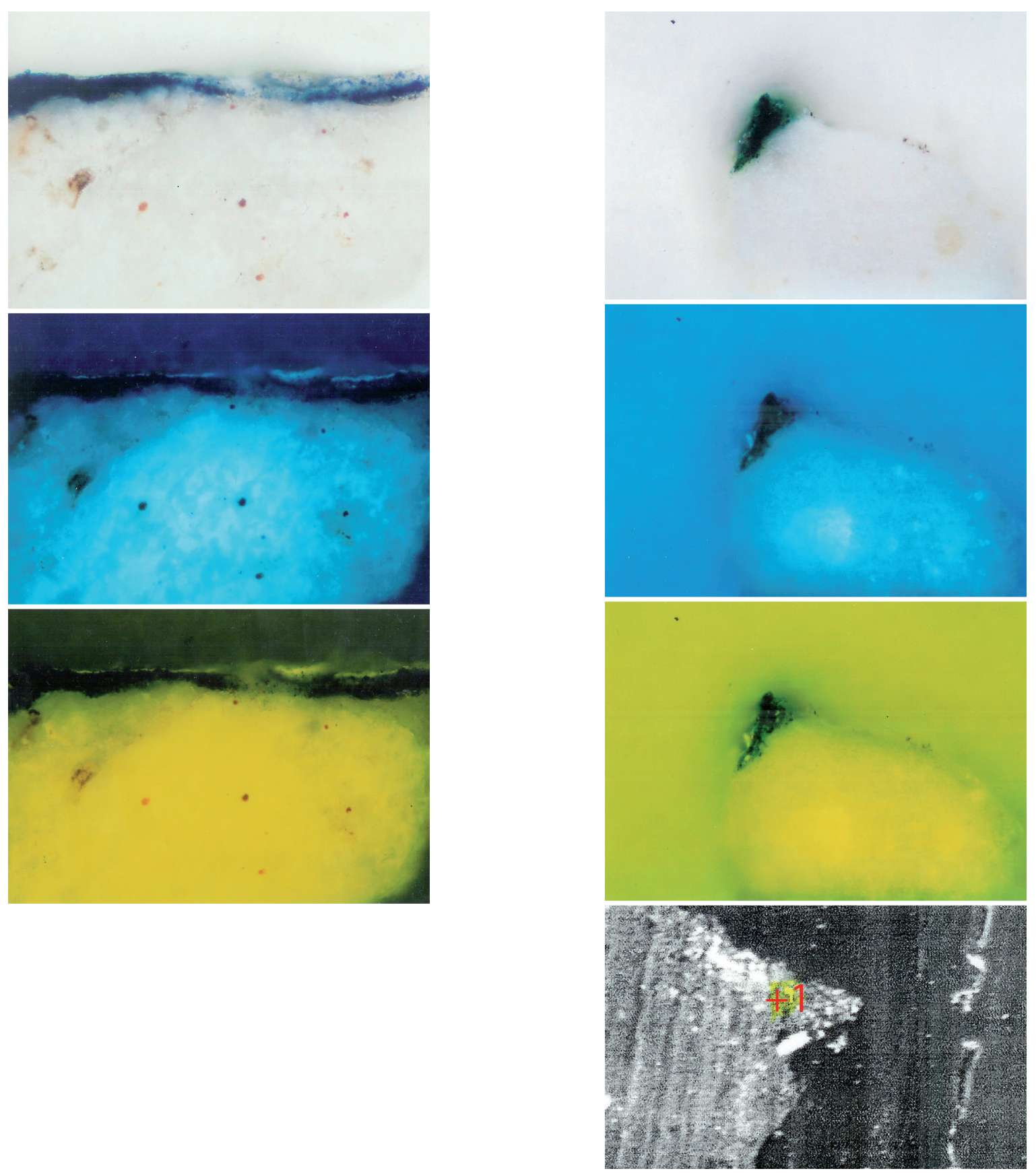

\begin{tabular}{cc}
\hline Oxide & $\mathbf{~ W t ~ [ \% ] ~}$ \\
\hline $\mathrm{MgO}$ & 1.47 \\
$\mathrm{Al}_{2} \mathrm{O}_{3}$ & 3.94 \\
$\mathrm{SiO}_{2}$ & 11.24 \\
$\mathrm{P}_{2} \mathrm{O}_{5}$ & 4.2 \\
$\mathrm{SO}_{3}$ & 16.77 \\
$\mathrm{~K}_{2} \mathrm{O}$ & 1.96 \\
$\mathrm{CaO}$ & 46.72 \\
$\mathrm{BaO}$ & 2.65 \\
$\mathrm{Cr}_{2} \mathrm{O}_{3}$ & 5.29 \\
$\mathrm{Fe}_{2} \mathrm{O}_{3}$ & 5.75 \\
$\mathrm{Total}$ & 100 \\
\hline
\end{tabular}

Figure 14. Sample 13; blue colour - artificial ultramarine, colouring earth, ochres, calcium sulphate.
Figure 15. Sample 14; green colour - chrome oxide, barium sulphate, colouring earth, ochres, calcium sulphate. 

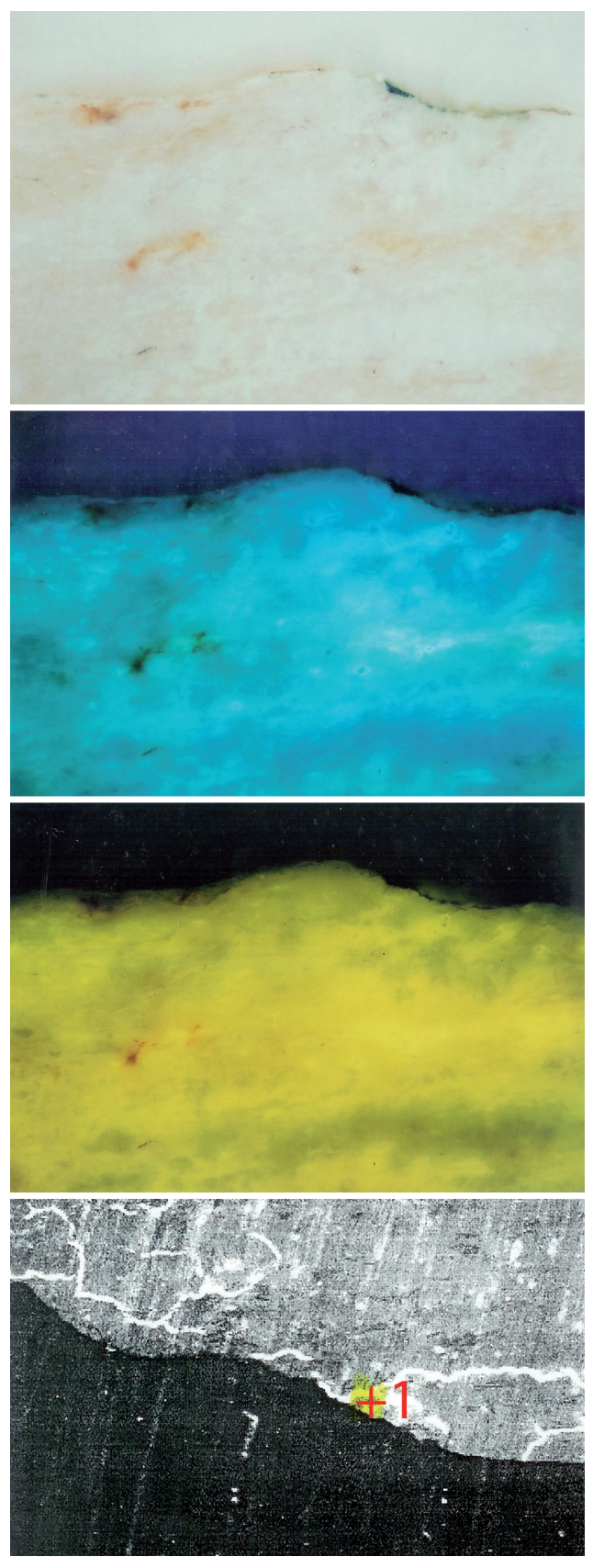

\begin{tabular}{cc}
\hline Oxide & Wt [\%] \\
\hline $\mathrm{MgO}$ & 2.39 \\
$\mathrm{Al}_{2} \mathrm{O}_{3}$ & 11.74 \\
$\mathrm{SiO}_{2}$ & 22.55 \\
$\mathrm{SO}_{3}$ & 17.09 \\
$\mathrm{~K}_{2} \mathrm{O}$ & 3.29 \\
$\mathrm{CaO}$ & 31.55 \\
$\mathrm{BaO}$ & 1.44 \\
$\mathrm{Cr}_{2} \mathrm{O}_{3}$ & 3.03 \\
$\mathrm{Fe}_{2} \mathrm{O}_{3}$ & 6.91 \\
$\mathrm{Total}$ & 100 \\
\hline
\end{tabular}

Figure 16. Sample 15; green colour - green earth, chrome oxide, calcium sulphate.

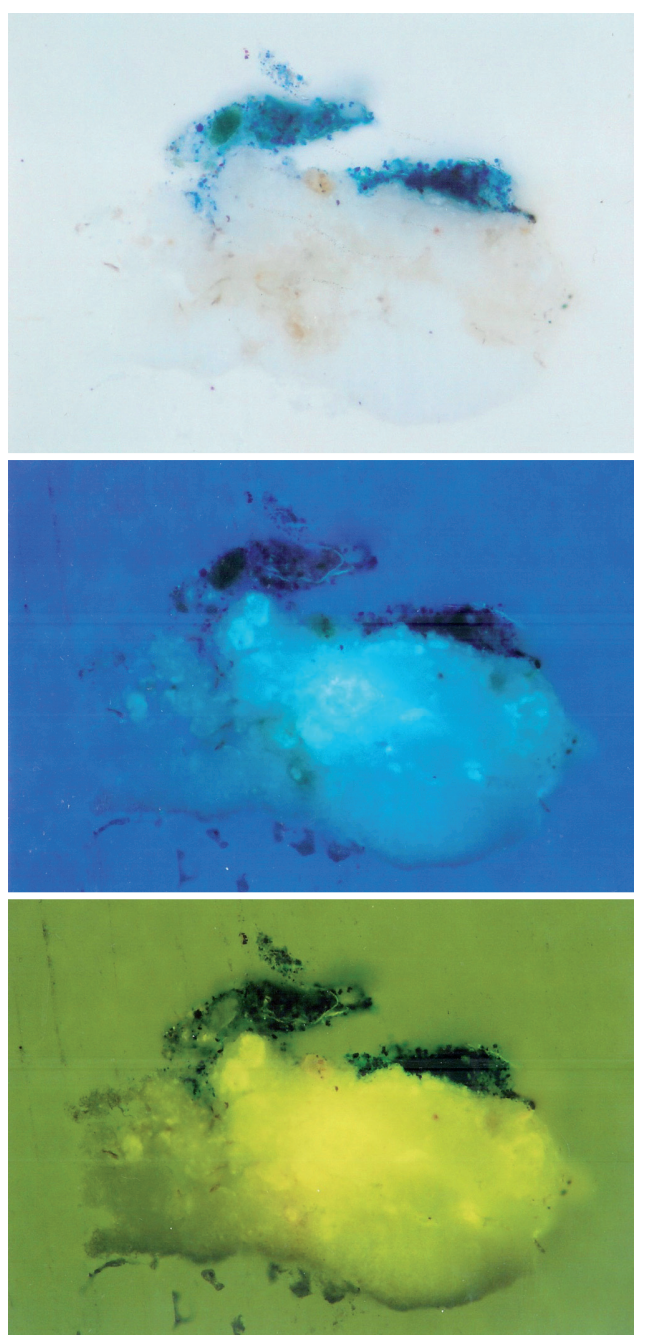

Figure 17. Sample 16; blue colour - artificial ultramarine, colouring earth, ochres, calcium sulphate. 


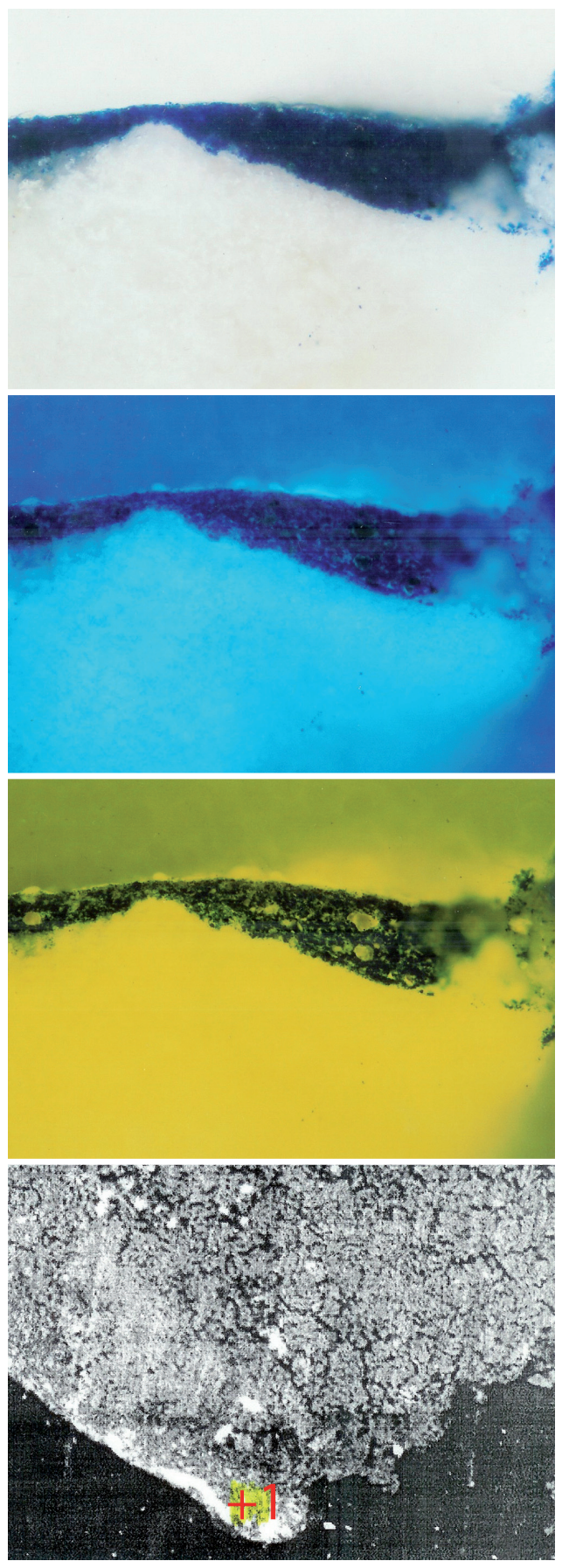

\begin{tabular}{cc}
\hline & $\mathbf{1}$ \\
Oxide & $\mathbf{W t}[\mathbf{\%}]$ \\
\hline $\mathrm{Na}_{2} \mathrm{O}$ & 9.85 \\
$\mathrm{MgO}$ & 2.03 \\
$\mathrm{Al}_{2} \mathrm{O}_{3}$ & 15.05 \\
$\mathrm{SiO}_{2}$ & 38.97 \\
$\mathrm{SO}_{3}$ & 18.91 \\
$\mathrm{~K}_{2} \mathrm{O}$ & 4.9 \\
$\mathrm{CaO}$ & 3.28 \\
$\mathrm{Fe}_{2} \mathrm{O}_{3}$ & 7.01 \\
$\mathrm{Total}$ & 100 \\
\hline
\end{tabular}

Figure 18. Sample 17; blue colour- artificial ultramarine, colouring earth, ochres, calcium sulphate.
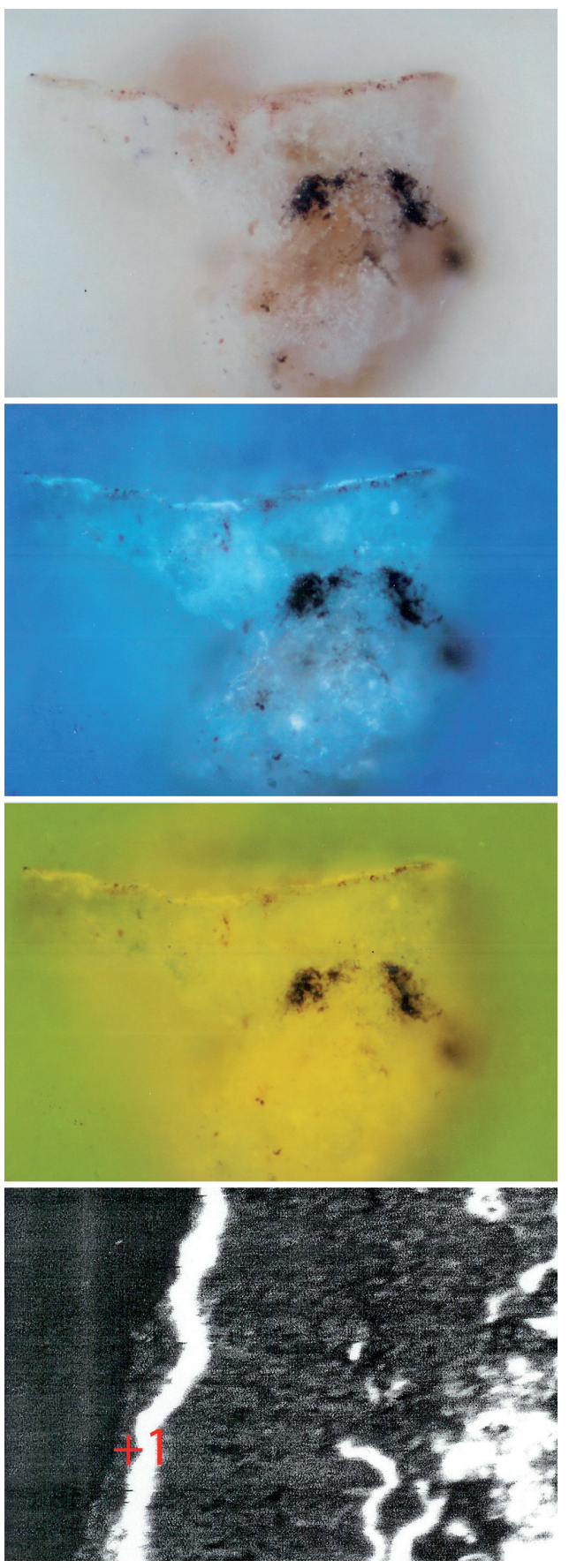

\begin{tabular}{cc}
\hline & $\mathbf{1}$ \\
Oxide & $\mathbf{W t}[\boldsymbol{\%}]$ \\
\hline $\mathrm{Na}_{2} \mathrm{O}$ & 1.99 \\
$\mathrm{MgO}$ & 3.52 \\
$\mathrm{Al}_{2} \mathrm{O}_{3}$ & 1.54 \\
$\mathrm{SiO}_{2}$ & 11.29 \\
$\mathrm{P}_{2} \mathrm{O}_{5}$ & 17.79 \\
$\mathrm{SO}_{3}$ & 11.67 \\
$\mathrm{~K}_{2} \mathrm{O}$ & 1.13 \\
$\mathrm{CaO}_{2}$ & 48.47 \\
$\mathrm{Fe}_{2} \mathrm{O}_{3}$ & 2.62 \\
$\mathrm{Total}$ & 100 \\
\hline
\end{tabular}

Figure 19. Sample 18; red colour - red ochre, colouring earth, calcium sulphate, zinc white. 

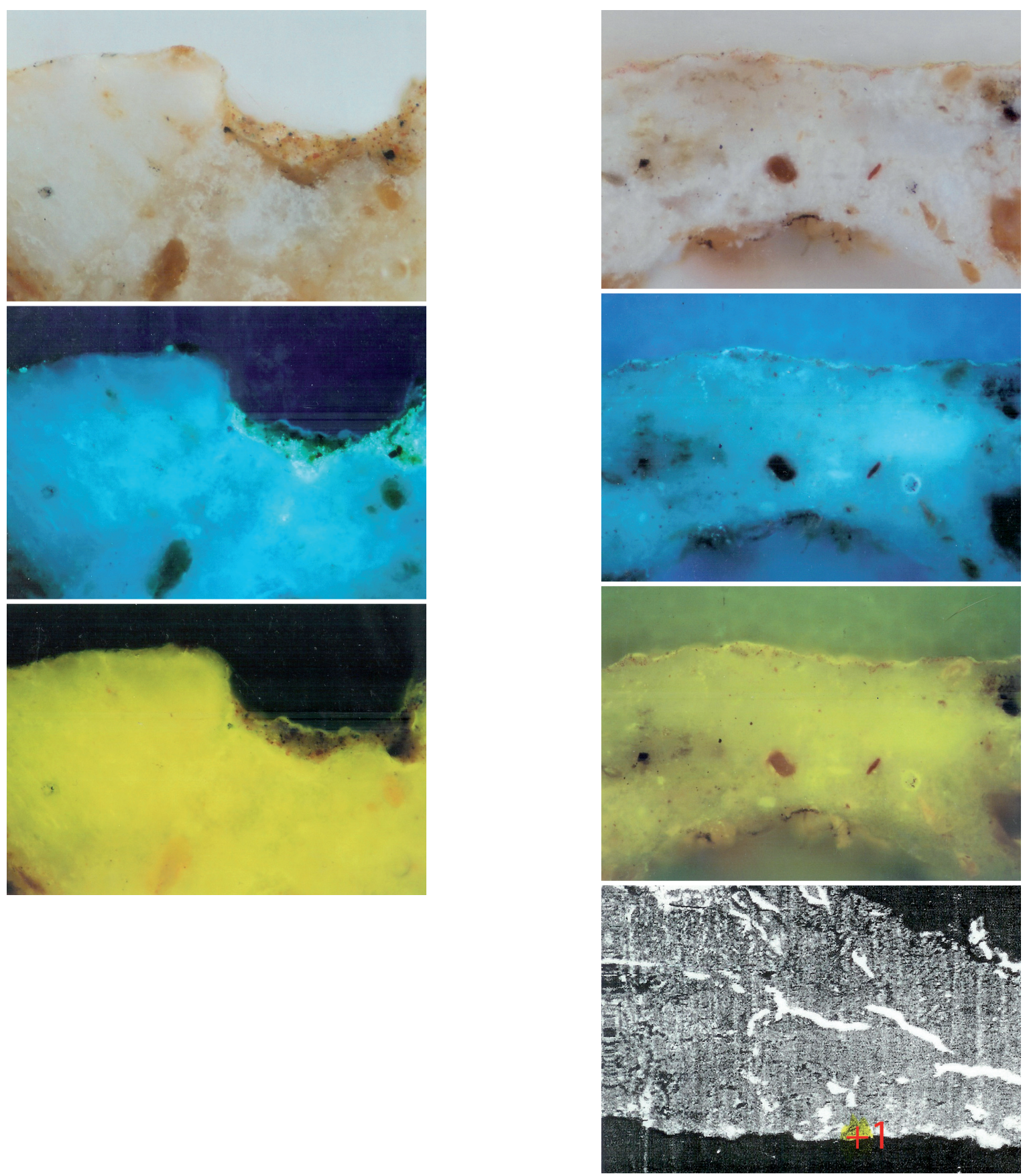

\begin{tabular}{cc}
\hline & $\mathbf{1}$ \\
Oxide & Wt [\%] \\
\hline $\mathrm{Na}_{2} \mathrm{O}$ & 1.05 \\
$\mathrm{MgO}$ & 0.7 \\
$\mathrm{Al}_{2} \mathrm{O}_{3}$ & 5.48 \\
$\mathrm{SiO}_{2}$ & 10.57 \\
$\mathrm{SO}_{3}$ & 27.89 \\
$\mathrm{~K}_{2} \mathrm{O}$ & 1.42 \\
$\mathrm{CaO}_{2}$ & 44.68 \\
$\mathrm{Cr}_{2} \mathrm{O}_{3}$ & 1.22 \\
$\mathrm{Fe}_{2} \mathrm{O}_{3}$ & 7.01 \\
$\mathrm{Total}$ & 100 \\
\hline
\end{tabular}

Figure 20. Sample 19; yellow colour - zinc white, colouring earth, ochres, calcium sulphate.
Figure 21. Sample 20; red colour - red ochre, colouring earth, calcium sulphate, chrome (green granule). 

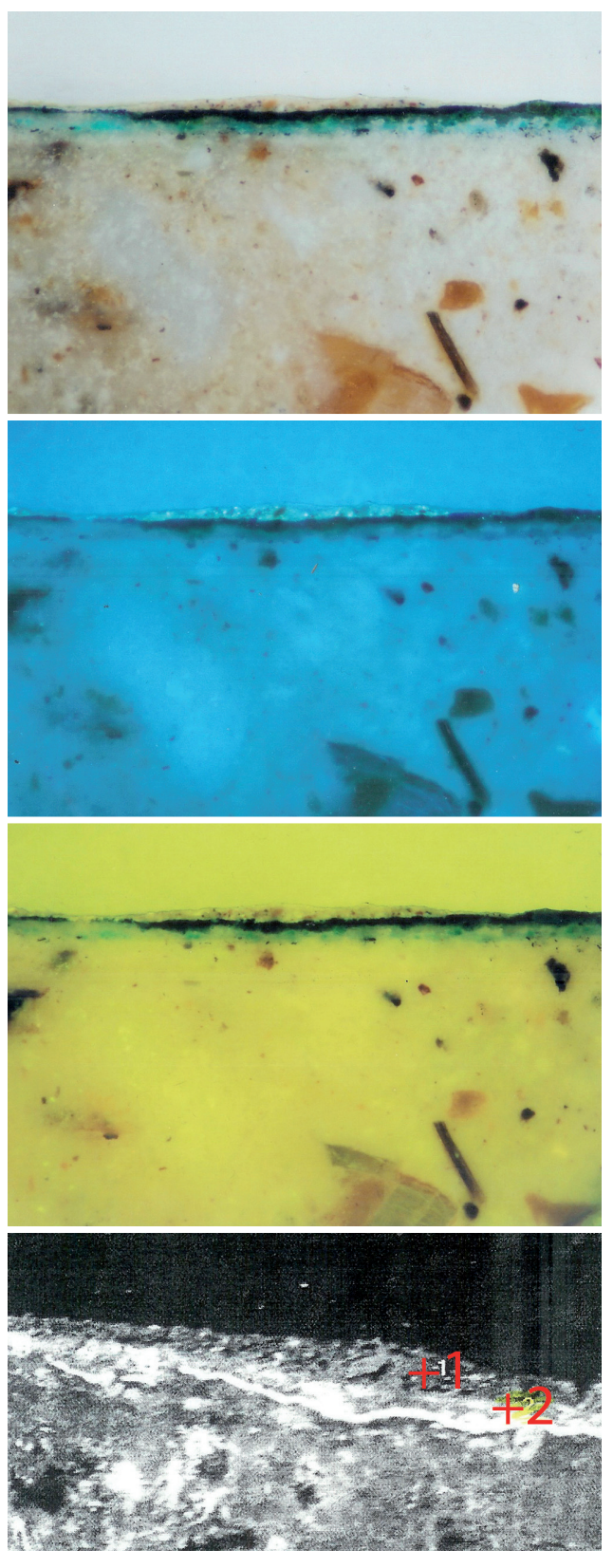

\begin{tabular}{cccc}
\hline & $\mathbf{1}$ & & \multicolumn{2}{c}{$\mathbf{2}$} \\
Oxide & $\mathbf{W t}[\mathbf{\%}]$ & Oxide & $\mathbf{W t}[\%]$ \\
\hline $\mathrm{MgO}$ & 1.52 & $\mathrm{Al}_{2} \mathrm{O}_{3}$ & 4.47 \\
$\mathrm{Al}_{2} \mathrm{O}_{3}$ & 15.62 & $\mathrm{SiO}_{2}$ & 9.52 \\
$\mathrm{SiO}_{2}$ & 24.13 & $\mathrm{SO}_{3}$ & 26.43 \\
$\mathrm{SO}_{3}$ & 7.64 & $\mathrm{~K}_{2} \mathrm{O}$ & 1.12 \\
$\mathrm{~K}_{2} \mathrm{O}$ & 1.87 & $\mathrm{CaO}$ & 15.56 \\
$\mathrm{CaO}$ & 13.14 & $\mathrm{BaO}$ & 6.01 \\
$\mathrm{TiO}_{2}$ & 17.31 & $\mathrm{Cr}_{2} \mathrm{O}_{3}$ & 0.85 \\
$\mathrm{Fe}_{2} \mathrm{O}_{3}$ & 6.5 & $\mathrm{Fe}_{2} \mathrm{O}_{3}$ & 1.12 \\
$\mathrm{ZnO}$ & 12.26 & $\mathrm{CuO}$ & 13 \\
& & $\mathrm{ZnO}$ & 6.9 \\
& & $\mathrm{As}_{2} \mathrm{O}_{3}$ & 15.02 \\
Total & 100 & $\mathrm{Total}$ & 100 \\
\hline
\end{tabular}

Figure 22. Sample 21; I - layer, ochre colour - titanium white and zinc white, colouring earth, ochres, calcium sulphate; II - lower layer, blue colour (with green) - artificial ultramarine; III - lower layer, green colour Schweinfurt green, chrome oxide, barium sulphate, colouring earth, ochres, zinc white, calcium sulphate.

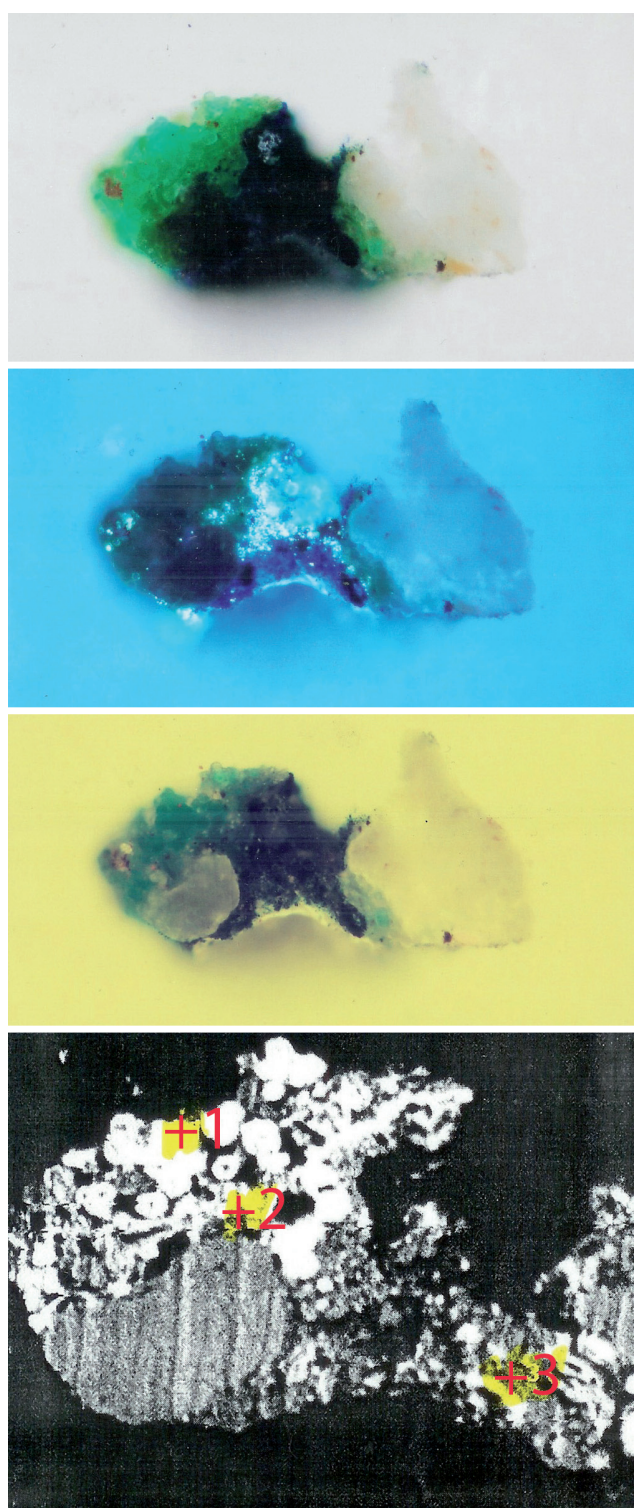

\begin{tabular}{|c|c|c|c|c|c|}
\hline \multicolumn{2}{|c|}{1} & \multicolumn{2}{|c|}{2} & \multicolumn{2}{|c|}{3} \\
\hline Oxide & Wt [\%] & Oxide & Wt [\%] & Oxide & Wt [\%] \\
\hline $\mathrm{CuO}$ & 33.8 & $\mathrm{Al}_{2} \mathrm{O}_{3}$ & 2.19 & $\mathrm{Al}_{2} \mathrm{O}_{3}$ & 20.53 \\
\hline \multirow[t]{11}{*}{$\mathrm{As}_{2} \mathrm{O}_{3}$} & 66.2 & $\mathrm{SiO}_{2}$ & 11.26 & $\mathrm{SiO}_{2}$ & 33.46 \\
\hline & & $\mathrm{P}_{2} \mathrm{O}_{5}$ & 1.47 & $\mathrm{SO}_{3}$ & 23.19 \\
\hline & & $\mathrm{SO}_{3}$ & 3.68 & $\mathrm{~K}_{2} \mathrm{O}$ & 2.58 \\
\hline & & $\mathrm{K}_{2} \mathrm{O}$ & 3.28 & $\mathrm{CaO}$ & 4.07 \\
\hline & & $\mathrm{CaO}$ & 5.45 & $\mathrm{Fe}_{2} \mathrm{O}_{3}$ & 0.99 \\
\hline & & $\mathrm{BaO}$ & 3.65 & $\mathrm{CuO}$ & 3.58 \\
\hline & & $\mathrm{Cr}_{2} \mathrm{O}_{3}$ & 5.17 & $\mathrm{ZnO}$ & 7.81 \\
\hline & & $\mathrm{Fe}_{2} \mathrm{O}_{3}$ & 11.42 & $\mathrm{As}_{2} \mathrm{O}_{3}$ & 3.8 \\
\hline & & $\mathrm{CuO}$ & 8.04 & & \\
\hline & & $\mathrm{ZnO}$ & 7.33 & & \\
\hline & & $\mathrm{PbO}_{2}$ & 37.07 & & \\
\hline Total & 100 & Total & 100 & Total & 100 \\
\hline
\end{tabular}

Figure 23. Sample 22; I - coating, green colour - Schweinfurt green, chrome oxide, barium sulphate, colouring earth, ochres, zinc white, slug, calcium sulphate; II - Lower layer, blue colour - artificial ultramarine, colouring earth, ochres, calcium sulphate. 

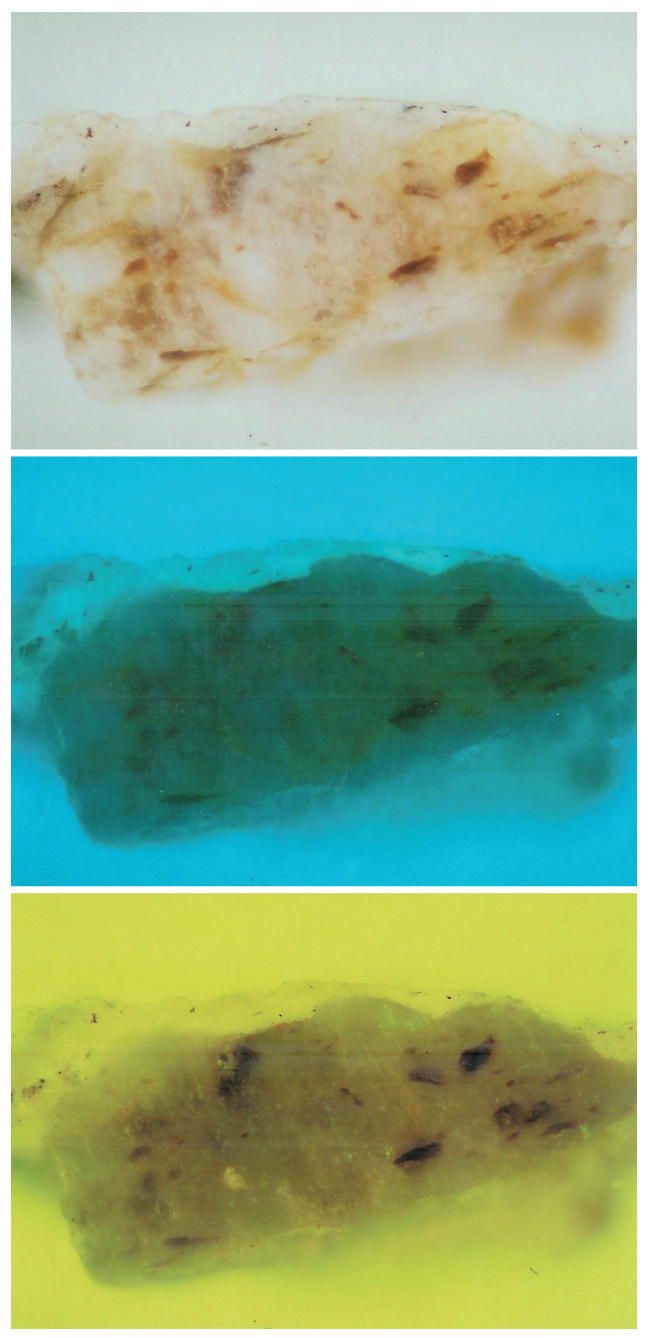

- Sample 4 (Figure 5): yellow colour - mars yellow, colouring earth, calcium sulphate.

- Sample 5 (Figure 6): red colour - red ochre, colouring earth, calcium sulphate, zinc white.

- Sample 6 (Figure 7): red colour - red ochre, colouring earth, calcium sulphate.

- Sample 7 (Figure 8): red colour - red ochre, colouring earth, calcium sulphate.

- Sample 8 (Figure 9): yellow colour - yellow and red ochres, colouring earth

- Sample 9 (Figure 10): green colour - green earth, chrome oxide (?), barium sulphate, colouring earth, ochres, calcium sulphate.

- Sample 10 (Figure 11): blue colour - artificial ultramarine, colouring earth, ochres, calcium sulphate.

- Sample 11 (Figure 12): I - coating, red colour - red ochre, colouring earth, calcium sulphate;

- II - lower coating, red colour - red ochre, colouring earth, cinnabar, chrome.

- Sample 12 (Figure 13): red colour - cinnabar.

- Sample 13 (Figure 14): blue colour - artificial ultramarine, colouring earth, ochres, calcium sulphate.

- Sample 14 (Figure 15): green colour - chrome oxide, barium sulphate, colouring earth, ochres, calcium sulphate.

- Sample 15 (Figure 16): green colour - green earth, chrome oxide, calcium sulphate

- Sample 16 (Figure 17): blue colour - artificial ultramarine, colouring earth, ochres, calcium sulphate.

- Sample 17 (Figure 18): blue colour - artificial ultramarine, colouring earth, ochres, calcium sulphate.

- Sample 18 (Figure 19): red colour-red ochre, colouring earth, calcium sulphate, zinc white.

- Sample 19 (Figure 20): yellow colour - zinc white, colouring earth, ochres, calcium sulphate.

- Sample 20 (Figure 21): red colour - red ochre, colouring earth, calcium sulphate, chrome (green granule).

- Sample 21 (Figure 22): I - layer, ochre colour - titanium white and zinc white, colouring earth, ochres, calcium sulphate; II - lower layer, blue colour (with green) artificial ultramarine; III - lower layer, green colour - Schweinfurt green, chrome oxide, barium sulphate, colouring earth, ochres, zinc white, calcium sulphate.

- Sample 22 (Figure 23): I - coating, green colour Schweinfurt green, chrome oxide, barium sulphate, colouring earth, ochres, zinc white, slug, calcium sulphate ; II - Lower layer, blue colour - artificial ultramarine, colouring earth, ochres, calcium sulphate.

- Sample 23 (Figure 24): white colour - white with green and yellow granules - colouring earth, ochres, calcium sulphate. 


\section{Conclusion}

The pigments found in the murals in the Rotunda of St. atherine in Znojmo lead to the following conclusions.

\subsection{Possibilities of the use during a certain historical era}

From discovered pigments, the lead white is used since Ancient era and it's therefore possible to identify it as an original pigment which was used by medieval painters. In the 19th century it was substituted by zinc white, which therefore could not be used by medieval painters. Zinc white was used in painting since 18th century but wasn't frequently present in art work before the second third of 19th century. The restorer Melicher used it for the restoration in the years 1891-1893 (see also Fišer 1949, 26). Meanwhile the natural ochres and clays were used by painters since the oldest times and therefore also in the rotund, the artificial ultramarine was offered on the market with painting pigments only since 1832 . A few years later, in 1838, the first transparent chromium oxide was produced, which was more frequently used only since 1859. Schweinfurt green, which was also found in the investigated paintings, was discovered only in 1800 , patented in 1814 and in 1920 its production was already banned due to its toxicity. An original medieval pigment is also green earth. This pigment is used in painting up to the present day, as well as minium (naturally occurring form of lead tetroxide used since Roman times). The last pigment which cannot be present in a medieval painting is Mars yellow whose production was first described in the middle of 19th century. For more detailed information about history of use of particular pigments see e.g. Nicolaus (2003), Schramm, Hering (1995), Slánský (1956), Šimůnková, Bayerová (1999), Šimůnková, Karhan (1993) or Wehlte (1990).

\subsection{The definition of original paintings and modern restorer's interventions}

From the pigment samples the following elements were identified as original: minium, green earth, yellow and brown ochres, and lead white. The original blue pigment was most probably blue ochre (vivianit), as has been already suggested by Fišer (1949). This pigment is present on paintings from the 12 th century onward.

Inauthentic pigments are present on murals in much larger quantity then experts previously supposed. The restoration processes that were organized in the Rotunda during the $19^{\text {th }}$ and $20^{\text {th }}$ centuries are the cause of their presence. During the $19^{\text {th }}$ century restoration work was done by T. Melicher. In the $20^{\text {th }}$ century it was done by F. Fišer and the restorers collective around A. Martan. The inauthentic pigments discovered in the surveys are: zinc and titanium white, transparent chromium oxide, Schweinfurt green and Mars yellow.

\section{Acknowledgments}

I would like to thank the Municipality of Znojmo, Ing. Marie Čejková, the South Moravian Museum of Znojmo, Director Durajková, the South Moravia Region Authority, the National Monument Institute, and Dr. Vítovský.

\section{References}

FIŠER, F. 1949: Zpráva o konservaci nástěnných maleb v kapli sv. Kateřiny ve Znojmě, 1947-1949. MS. Deposited: Archiv of the National Heritage Institute in Brno, Brno.

MERHAUTOVÁ, A., TřEŠTÍK, D. 1984: Románské uměni v Čechách a na Moravě. Praha.

NICOLAUS, K. 2003: DuMonts Handbuch der Gemäldekunde, Gemälde erkennen und bestimmen. Cologne.

SCHRAMM, H. P., HERING, B. 1995: Historische Malmaterialien und ihre Identifizierung. Stuttgart.

SLÁNSKÝ, B. 1956: Technika malby. Praha.

ŠIMŮNKOVÁ, E., BAYEROVÁ, T. 1999: Pigmenty. STOP, Praha.

ŠIMU゚MKOVÁ, E., KARHAN J. 1993: Pigmenty, barviva a metody jejich identifikace. Praha.

TRAPP, M. 1862: Die Wandgemelde in der Kapelle (Heidentempel) der Margrafenburg zu Znaim. Brünn.

WEHLTE, K. 1990: Wekstoffe und Techniken der Malerei. Ravensburg. 